WENHONG LI

CONG WANG

SHAN ZHANG

and

MINGJUN XU

\title{
Fault line selection research of single phase grounding circuit based on improved Hilbert-Huang transform
}

For single-phase ground fault in resonant grounded power supply system, it is difficult to detect the fault line, while the existing methods have the shortcomings such as slow speed and large amount of computation. In our work the system natural oscillation wave contained in the zero sequence current is found to have opposite polarity and greater amplitude in fault line compared to that in non-fault line, and is proved to have a corresponding relationship with the first intrinsic mode function (IMF1) extracted from the same zero sequence current by empirical mode decomposition (EMD) of (Hilbert-Huang transform (HHT). Therefore, a new method for determining the single-phase ground fault line is proposed by finding the IMF1, extracted from the zero sequence current of each line by EMD of HHT, with the largest amplitude and opposite polarity compared to that of other lines. Meanwhile, EMD algorithm is improved to further enhance the fault detection rate based on the system natural oscillation wave's frequency and its attenuation trend in resonant grounding system. Simulation experiment and real data analysis indicate that the mentioned method is correct and fast.

Keywords: Resonant grounding system, zero sequence current, HHT, EMD, fault line selection

\subsection{Introduction}

A ccording to the provisions of the national coal mine safety regulations, several voltage levels such as $10 \mathrm{kV}, 35 \mathrm{kV}$ and $66 \mathrm{kV}$ are adopted in large and medium sized mine power supply, and their operation mode of neutral point via arc suppression coil is referred to as small current grounded (resonant grounding system) power supply network. When single-phase grounding and short-circuit fault occur in the power supply network, the inductance current of arc suppression coil will compensate capacitance current working in system [1], which will make the residual fault

Messrs. Wenhong Li, Shan Zhang, Department of Electrical Engineering and Information Technology, University of Science and Technology, Jinan, Cong Wang and Mingjun Xu, College of Electrical Engineering and Automation, University of Science and Technology, Qingdao, China. current small and the electric arc suppressed, and will not cause the switch trip. Then the system can continue to run from 1 to $2 h[2]$. However, when single-phase grounding fault occurs, non fault line's voltage is 3 times of the original tick due to that the single-phase grounding is asymmetric fault. If the fault line is not removed timely, it may further develop into two-phase short circuit grounding fault, which will cause large area blackout and other accidents, and seriously affect the safe production and personal safety in coal mine [3]. To select the fault line quickly and to remove it timely are the keys to ensure the safety of coal mine production and the workers.

With the extensive application of large power inverter and reactive power compensation device, the mine power network contains various harmonics and spurious waveform, which makes the small fault signal in the single phase grounding fault such as the zero sequence current suffer the serious disturbance and makes the fault line selection become more difficult. Steady-state and transient line selection is the main research method, among them the methods such as zero sequence current amplitude comparison method, zero sequence current phase comparison method, amplitude and phase comparison method, are based on the steady component, which need long time to obtain fault signal and cannot identify the first fault line quickly in the actual application, and often cause wrong selection. The other methods such as first half wave method, energy method, wavelet analysis method, travelling wave method are based on the transient component [4-5]. The wavelet analysis method [6], especially the multi-scale wavelet analysis method has the characteristics of instantaneous frequency analysis, which can find fault line [7] in 1/2 cycles. But the wavelet transform needs to prechoose wavelet function and its analytic ability depends on the choice of base function and threshold, thus its adaptability is not strong and the operation process is long. Therefore, it is not conducive to use in the field application. Hilbert-Huang transform (HHT) is a timefrequency analysis method for transient signal, following after wavelet analysis, whose advantage is that it can select the base function to decompose signal according to its own function characteristics. So it can be used to analyze 
instantaneous frequency [8-9]. But the EMD of HHT has the problems such as long analysis time and the end effect.

This paper analyzes the zero sequence current caused by single-phase ground fault in resonant grounded power supply system and expresses it as a sum of different frequency signals. Then the relationship between these different frequency signals and the intrinsic mode functions extracted from the same zero sequence current by EMD of HHT are explored. Finally, a new method for determining the singlephase ground fault line is proposed.

\subsection{Zero sequence current caused by single-phase grounding fault in resonant grounding system}

Fig.1 shows the resonant grounding system in mine high voltage power supply network, where the Petersen coil grounding mode L (resonant grounding operation) is used. As shown in Fig.1, there are 3 loaded lines, and $\mathrm{C}_{01}, \mathrm{C}_{02}, \mathrm{C}_{03}$, represent each line capacitance to ground respectively, and IL represents arc suppression coil current. In addition, one arrow line in Fig. 1 represents the direction of the capacitive grounding current in the line. When the circuit is running normally, the compatible current of each phase of each line varies with the line voltage. However, these current phases of each line are different, so the sum of three phase current vector of each line, called zero sequence current, is zero. But when $\mathrm{C}$ phase ground fault of line 3 in the resonant grounding system occurs as indicated in Fig.1, the zero sequence current of each line is not zero due to the asymmetry of the system. Furthermore, there are no ground current in $\mathrm{C}$ phase of line 1 and 2, while the $\mathrm{C}$ phase

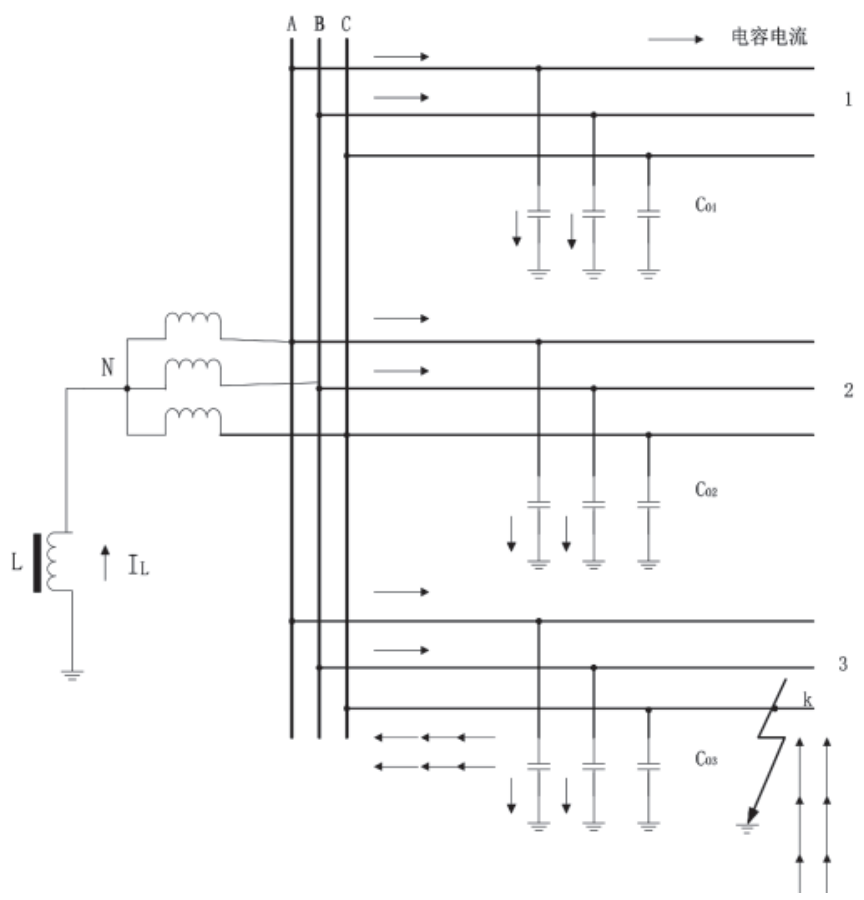

Fig.1 Single-phase grounding fault current distribution of resonant grounding system grounding current of line 3 exists, that is, its value is equal to the sum of A and B phase current of all lines, and its direction is opposite to the sum vector. Therefore, the zero sequence current of line 3 is the sum of $\mathrm{A}$ and $\mathrm{B}$ phase grounding currents in all feeder lines under the bus line, while the zero sequence current of line 1 or 2 is the sum of $A$ and B phase grounding currents in each own line, having smaller value and opposite direction compared to the former one. Thus based on the above principle, the fault line can be determined by detecting the zero sequence current of each line [1]. In practice, the acquired zero sequence current signals contain a variety of voltage signals and attenuates gradually, so it cannot be used to select the fault line directly.

According to the description of the literature [1], the transient grounding current of single phase grounding can be expressed as Equation (1):

$$
\begin{aligned}
i_{d} & =\left(I_{C m}-I_{L m}\right) \cos (\omega t+\varphi)+ \\
& I_{C m}\left(\frac{\omega_{f}}{\omega} \sin \varphi \sin \omega t-\cos \varphi \cos \omega_{f} t\right) e^{-\frac{t}{\tau_{C}}} \\
& +I_{L m} \cos \varphi e^{-\frac{t}{\tau_{L}}}
\end{aligned}
$$

where,

$i_{d}$ : Zero sequence transient grounding current of fault phase

$I_{c m}$ : The sum of the capacitor current amplitude of each phase of all feeder lines

$I_{L m}$ : The compensation current amplitude of the circuit inductance and arc suppression coil when the load is unbalanced or neutral point shift or fault

$\omega$ : Alternating current waveform frequency $50 \mathrm{~Hz}$

$\varphi$ : Phase of sine voltage when the fault phase is grounded

$\omega_{f}$ : Natural oscillation frequency of resonant grounding system, determined by the parameters of the circuit resistance, inductance, capacitance and arc suppression coil in the resonant grounding system, 40 100 times of $50 \mathrm{~Hz}$ frequency, generally relatively high.

Then in the system as shown in Fig.1, we can deduce the zero sequence current of the fault line and non-fault line as Equation (2) and (3) respectively.

$$
\begin{aligned}
i_{0}= & \left(\sum_{i=1}^{n-1} 2 I_{C m i}-I_{L m}\right) \cos (\omega \boldsymbol{t}+\varphi)+ \\
& \sum_{i=1}^{n-1} 2 I_{C m i}\left(\frac{\omega_{f}}{\omega} \sin \varphi \sin \omega t-\cos \varphi \cos \omega_{f} t\right) e^{-\frac{t}{\tau_{C}}} \\
& +I_{L m} \cos \varphi e^{-\frac{1}{\tau_{L}}} \\
i_{0}= & \left(-2 I_{C m}-I_{L m}\right) \cos (\omega t+\varphi)+ \\
& \left(-2 I_{C m}\right)\left(\frac{\omega_{f}}{\omega} \sin \varphi \sin \omega t-\cos \varphi \cos \omega_{f} t\right) e^{-\frac{t}{\tau_{C}}} \\
& +I_{L m} \cos \varphi e^{-\frac{1}{\tau_{L}}}
\end{aligned}
$$

We analyze the Equation (2) and further decompose it as 
Equation (4) on the basis of the combination of similar waveform:

$$
\begin{aligned}
i_{0}= & \left(\sum_{i=1}^{n-1} 2 I_{C m i}-I_{L m}\right) \cos (\omega t+\varphi)+ \\
& \sum_{i=1}^{n-1} 2 I_{C m i}\left(\frac{\omega_{f}}{\omega} \sin \varphi \sin \omega t\right) e^{-\frac{1}{T_{C}}}+ \\
& \sum_{i=1}^{n-1} 2 I_{C m i}\left(\cos \varphi \cos \omega_{f} t\right) e^{-\frac{t}{T_{C}}}+I_{L m} \cos \varphi e^{-\frac{t}{T_{L}}}
\end{aligned}
$$

Then we can see from Equation (4) as follows. The first term in the Equation (4) is the $50 \mathrm{~Hz}$ fundamental wave signal with stable amplitude, which always exists after the ground fault occured. The second term in the Equation (4) is a $50 \mathrm{~Hz}$ signal whose amplitude decays exponentially. The third term in the Equation (4) is the high frequency oscillation signal whose frequency is $\omega_{f}$ and amplitude decays exponentially. The fourth term in the Equation (4) is the monotonic signal and attenuates gradually. The amplitude of the third and four items in the Equation (4) is related to the phase $\varphi$ of the fault. Namely, when the fault occurs in the 0 degree phase of sine voltage, the value of phase current achieves the maximum, when the fault occurs in \pm 90 degree phase of sine voltage, the phase current is zero. However, in practice, the single-phase grounding fault occurs in the early phase of $0 \sim 65$ degrees [13].

As we know, wavelet multi-scale analysis [10] is based on the principle that the sum of modulus values of fault line and non-fault line in a period of time exists opposite polarity or maximum's character. Fundamental wave method is based on the principle that the fundamental waves contained in the zero sequence current of the fault line and non-fault line have the characteristic of opposite polarity which can be seen from the Equations (2) and (3). However, the first and second items in the Equation (4) have the same frequency, which makes the second term have an effect on the first iterm before the second term completely decay. Therefore, the sampling data must be collected after the 30 milliseconds of the fault occurrence time, which causes that fault line selection costs a long time.

We analyze the Equation (4) and find its third term can be used for fault line detection due to the following reasons. Firstly, the third term has opposite polarity and greater amplitude in fault line compared to that in non-fault line, which makes it easier to be used to detect the fault line. Secondly, the signal frequency of the third term is very high, which makes that the time for sampling several cycles data is very short and the detection speed is fast. Then the key is how to extract the third item of Equation (4) from the zero sequence current signas. Therefore, we study the HHT, a time-frequency analysis method for transient signal, and try to find the relationship between the many kinds of frequency signals contained in the zero sequence current and the intrinsic mode functions extracted from the same zero sequence current by EMD of HHT.

\subsection{HHT of the zero sequence current}

The HHT mainly consists of two parts, that is, the first part is the empirical mode decomposition (EMD) proposed by Huang, which is used to decompose the signal into a number of specific components, called intrinsic mode function (IMF), and the second part is the Hilbert transform for each IMF.

\subsection{EMPIRICAL MODE DECOMPOSITION}

In HHT, the IMF extracted by EMD contains the component of the signal characteristic which plays a very important role that IMF characteristics determine whether the original signal can be described in the time-frequency domain after it is transformed by Hilbert. Therefore, the IMF must meet the following criteria:

1. Assuming that the total number of extreme values of the signal is $m$ and the number of zero-crossing points is $n,|m-n|<1$.

2. At any point in the signal, the envelope of the local extreme point fitting is symmetrical about the time axis.

The decomposition process of EMD is a cyclic screening process and each IMF has different characteristics, thus the original signal is changed into a sum of a variety of fluctuation series with different characteristics.

Specific steps of EMD are as follows:

1. Cubic spline curve fitting method is used to connect the local maximum values of the original signal to the upper envelope curve $U_{x}(t)$, in the same way the local minimum values of the original signal are connected to the lower envelope curve $\mathrm{V}_{x}(\mathrm{t})$.

2. Calculate the average value $m_{1}(\mathrm{t})$ :

$m_{1}(t)=\frac{U_{x}(t)+V_{x}(\mathrm{t})}{2}$

3. Get the IMF:

$h_{1}(t)=x(t)-m_{1}(t)$

According to the Equation (6), $h_{1}(\mathrm{t})$ can be got. If the $h_{1}(\mathrm{t})$ can meet the IMF conditions, it is the IMF1 of the original signal, otherwise, the signal $x(\mathrm{t})$ is replaced by $h_{1}(\mathrm{t})$, and the first two steps are repeated until the $h_{1}(\mathrm{t})$ can meet the conditions of the IMF component, then the $h_{1}(\mathrm{t})$ is the first IMF component, $c_{1}(\mathrm{t})$, as expressed in Equation (7).

$$
c_{1}(t)=h_{1}(t)
$$

(4) Get the residual component $r_{1}(\mathrm{t})$ as expressed in Equation (8)

$$
r_{1}(t)=x(t)-c_{1}(t)
$$

(5) Order $r_{1}(\mathrm{t})$ as $x(\mathrm{t})$, the loop from step (1) to (4) executes again. After executing $\mathrm{n}$ times, we can get $n$ IMF components. When $r_{n}(\mathrm{t})$ is a constant or a monotonic function, the EMD algorithm is stopped. 
Then the original signal $x(\mathrm{t})$, namely, the zero sequence current, can be expressed as:

$$
x(t)=\sum_{i=1}^{n} c_{i}+r_{n}
$$

3.2 Hilbert transform AND PhySiCAl MEANING OF IMF OF THE ZERO SEQUENCE CURRENT

After the original signal $x(\mathrm{t})$, namely, the zero sequence current, is decomposed by EMD, the obtained IMF component $c_{i}(\mathrm{t})$ is transformed by Hilbert as expressed in Equation (10).

$$
\hat{c}_{i}(t)=\frac{1}{\pi} \int_{-\infty}^{+\infty} \frac{\mathrm{c}_{i}(\tau)}{t-\tau} d \tau
$$
(11)

Then the analytic signal can be constructed as Equation

$$
z_{i}(t)=c_{i}(t)+j \hat{c}_{i}(t)=a_{i}(t) e^{j \phi_{i}(t)}
$$

And the amplitude function of the analytic signal is expressed as Equation (12),

$$
a_{i}(t)=\sqrt{c_{i}^{2}(t)+\hat{c}_{i}^{2}(t)}
$$

The corresponding phase function is expressed as Equation (13).

$$
\Phi_{i}(t)=\arctan \frac{\hat{c}_{i}(t)}{c_{i}(t)}
$$

Then function derivation of Equation (13) can be obtained as expressed in Equation (14), which is consistent with the definition of the frequency, thus defined as instantaneous frequency

$$
\omega_{i}(t)=\frac{d \Phi_{i}(t)}{d t}
$$

Then the original signal $x(\mathrm{t})$, namely, the zero sequence current, can be expressed as Equation (15) under the condition of omitting $r n(t)$.

$$
x(t)=\operatorname{Re} \sum_{i=1}^{n} a_{i}(t) e^{j \Phi_{i}(t)}=\operatorname{Re} \sum_{i=1}^{n} a_{i}(t) e^{j \int \omega_{i}(t) d t}
$$

Therefore, Equations (15) and (9) indicate the same signal, namely, the zero sequence current, which illustrates that the IMF components in Equation (9) has a corresponding relationship to the real part of the complex consisted of $a_{i}(\mathrm{t})$ and $\omega_{i}(\mathrm{t})$ in Equation (15), which also indicates that the signal obtained by HHT contains the instantaneous frequency of the original signal and many kinds of frequency waves are separated by EMD of HHT. In the meantime, Equation (4) indicates that the zero sequence current consists of a variety of frequency waves such as high frequency oscillation signal with big amplitude, the harmonic signal with frequency coming second and low amplitude, the $50 \mathrm{~Hz}$ fundamental wave with a longer duration and stable amplitude, and nonlinear single stable signal. All above illustrate that there are corresponding relationship between the various frequency signals contained in the zero sequence current and the intrinsic mode functions extracted from the same zero sequence current by EMD of HHT, namely, system natural oscillation wave contained in the zero sequence current has a corresponding relationship with the first intrinsic mode function (IMF1) extracted from the same zero sequence current by EMD of HHT, IMF2 6 are corresponding with each order harmonic signal, IMF7 and residual signal are corresponding with the fundamental wave. Therefore, it can be concluded that we can obtain the third item of Equation (4) from the zero sequence current signal by extracting the IMF1 from the same zero sequence current through EMD of HHT.

\subsection{IMPROVEMENT OF EMD}

We analyze the EMD and find that each order IMF is obtained by calculating the envelope curve of the original signal for several dozens of times, and the envelope curves are the maximum envelope of the original signal, which are fitted by the extreme points of the original signal segmentation based on cubic spline interpolation function as Equation (16). And each time of the fitting needs to be completed by the process of initial value setting, step length calculation and iteration step by step, especially, three times polynomial is used as the spline interpolation function, the initial value is set before each fitting, and the number of the sample data is not clearly defined, which makes the envelope calculated by EMD meet the IMF condition, and makes EMD have the adaptability to any signal. But the disadvantage of it is that the amount of computation is too large.

$$
s_{i}=a_{i} l^{3}+b_{i} l^{2}+c_{i} l+d_{i},
$$

where,

$S_{i}$ : Cubic spline interpolation function fitted by continuous multiple extreme points

$a_{i}, b_{i}, c_{i}, d_{i}:$ Coefficients of the cubic spline interpolation function, need to be solved

\section{$l$ : Sample serial number, data sampling time}

\section{S(n): Fitting sample data sequence}

However, the third item in Equation (4) has the characteristics of stable oscillation period and extreme points with exponential decay trend; then based on this, we can find the extreme points quickly as the following method.

Firstly, in each period $\mathrm{Ti}$, there is one maximum point and one minimum point, then which can be used to calculate the number of fitting as $\mathrm{n}=\mathrm{T}_{0} / \mathrm{T}_{\mathrm{i}}$. where,

$T_{0}$ : Zero sequence current signal sampling time

$T_{i}$ : Respectively is the line oscillation cycle (the reciprocal of the oscillation frequency), the harmonic cycle, $20 \mathrm{~ms}$, etc.

Secondly, because, in the zero sequence current, the amplitude of system natural oscillation wave is monotonic 
and fast attenuation, harmonic signal is periodic, and fundamental wave is sinusoidal periodic signal, thus the initial value of coefficients of the cubic spline interpolation function can be set as monotone attenuation signal, periodic signal and sinusoidal periodic signal respectively. Therefore, the improved algorithm process of EMD is shown as in Fig.2.

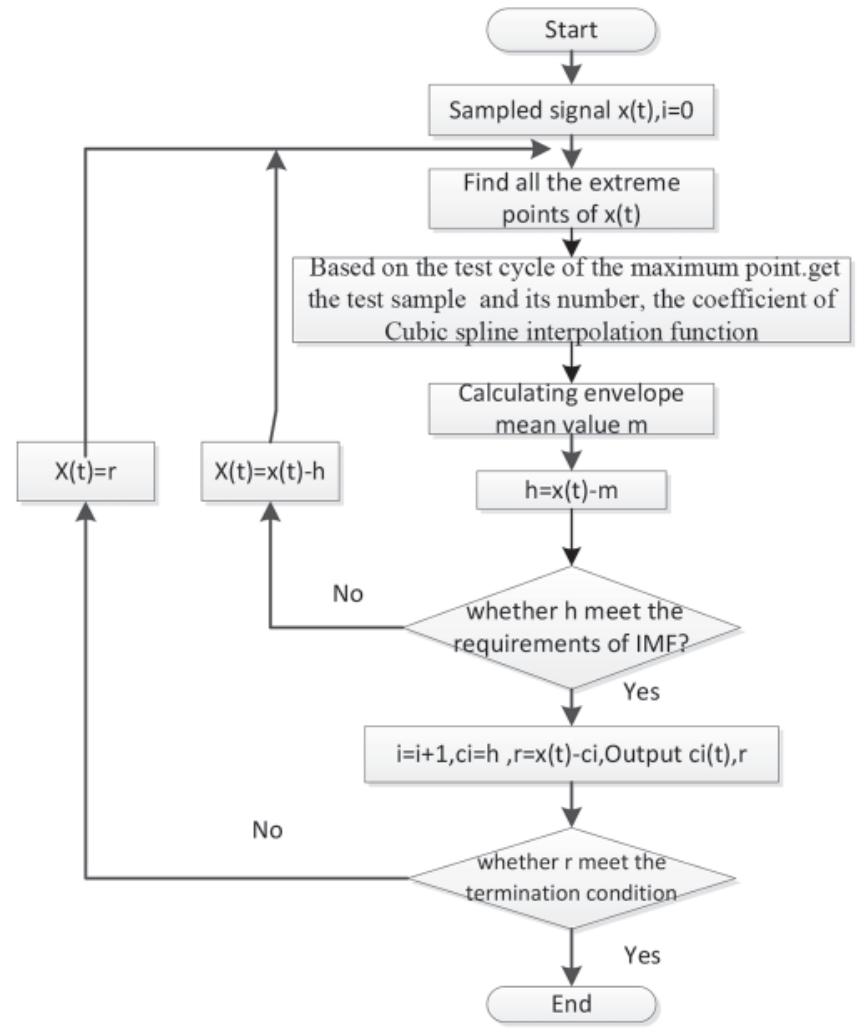

Fig.2 Flow chart of improved EMD

In all, through the above research and analysis, the system natural oscillation wave contained in the zero sequence current is found to have opposite polarity and greater amplitude in fault line compared to that in non-fault line, and is proved to have a corresponding relationship with the IMF1 extracted from the same zero sequence current by EMD of HHT. Therefore, we can determine the single-phase ground fault line by finding the IMF1, extracted from the zero sequence current of each line by EMD of HHT, with the largest amplitude and opposite polarity compared to that of other lines.

\subsection{Experiment}

According to the above mentioned method for fault line selection, we carry out the simulation experiment and real data analysis, and the boundary of EMD is dealt with the method of zero padding [15].

\subsection{EXPERIMENT SCHEME OF FAULT LINE DETECTION METHOD}

1. The zero sequence current sensor is installed at the end of each feeder line near the bus terminal, and the zero sequence current of each line is collected in real time with the sampling rate of $100 \mathrm{Ksa} / \mathrm{s}$. When the zero sequence current signals exceed a threshold value, the data is stored in 0.05 seconds before and after the threshold.

2. 4000th points to 9000th points of the saved data are captured. And according to the natural frequency of the system, the maximum point and the minimum point are determined, exponential decay rate is selected for the initial value of the cubic spline interpolation function. Then the IMF 1 of the zero sequence current of each line is calculated according to the process shown in Fig. 2.

3. 500-1500 points signal of IMF1 of each line are compared, and we can determine the fault line whose IMF1 has the largest amplitude and opposite polarity compared to that of other lines.

\subsection{SiMULATION EXPERIMENT}

A $10 \mathrm{kV}$ resonant grounding system is built by use of MATLAB/SIMULINK, and the power distribution system has three outlets which are used to simulate 10kmYGv22-10-3*185 cables and whose load is $1200 \mathrm{kVA}, 600 \mathrm{kVA}, 2400 \mathrm{k}$ VA respectively. Then the fault point is located at the $1 \mathrm{~km}$ of outlet 3 as shown in Fig.3. The sampling period is $\mathrm{T}_{\mathrm{s}}=10^{-5} \mathrm{~s}$.

The zero sequence current of line 1 is shown in Fig.3, and that of line 2 is similar to Fig. 4 . The zero sequence current of fault line 3 is shown in Fig. 5.

The IMF1 of non-fault line and fault line is shown in Figs. 6 and 7 respectively, which is calculated according to the improved DMT of HHT and illustrates that the IMF1 of fault line has the largest amplitude and opposite polarity compared to that of other non-fault lines. And the former 20 data of IMF1 from three lines are listed in Table 1.

Table 1: Partial data of IMF1 from three lines

\begin{tabular}{cccc}
\hline Order & First Line & Second line & Third line \\
\hline 1 & -265.1 & -155.5 & 424.16 \\
2 & -266.4 & -160.08 & 428.12 \\
3 & -108.35 & -67.227 & 175.55 \\
4 & 153.71 & 92.944 & -247.82 \\
5 & 265.27 & 159.19 & -425.99 \\
6 & 212.31 & 126.16 & -339.53 \\
7 & 46.212 & 25.933 & -72.605 \\
8 & -141.42 & -85.994 & 227.61 \\
9 & -260.28 & -155.33 & 416.66 \\
10 & -259.04 & -151.64 & 412.57 \\
11 & -143.68 & -81.529 & 226.78 \\
12 & 29.514 & 20.435 & -49.399 \\
13 & 182.38 & 108.94 & -291.58 \\
14 & 248.89 & 146.87 & -396.05 \\
15 & 202.31 & 119.3 & -321.87 \\
16 & 66.898 & 40.169 & -107.4 \\
17 & -95.334 & -54.542 & 149.46 \\
18 & -212.19 & -122.88 & 334.63 \\
19 & -232.68 & -135.59 & 367.94 \\
20 & -149.53 & -88.869 & 238.37 \\
\hline
\end{tabular}




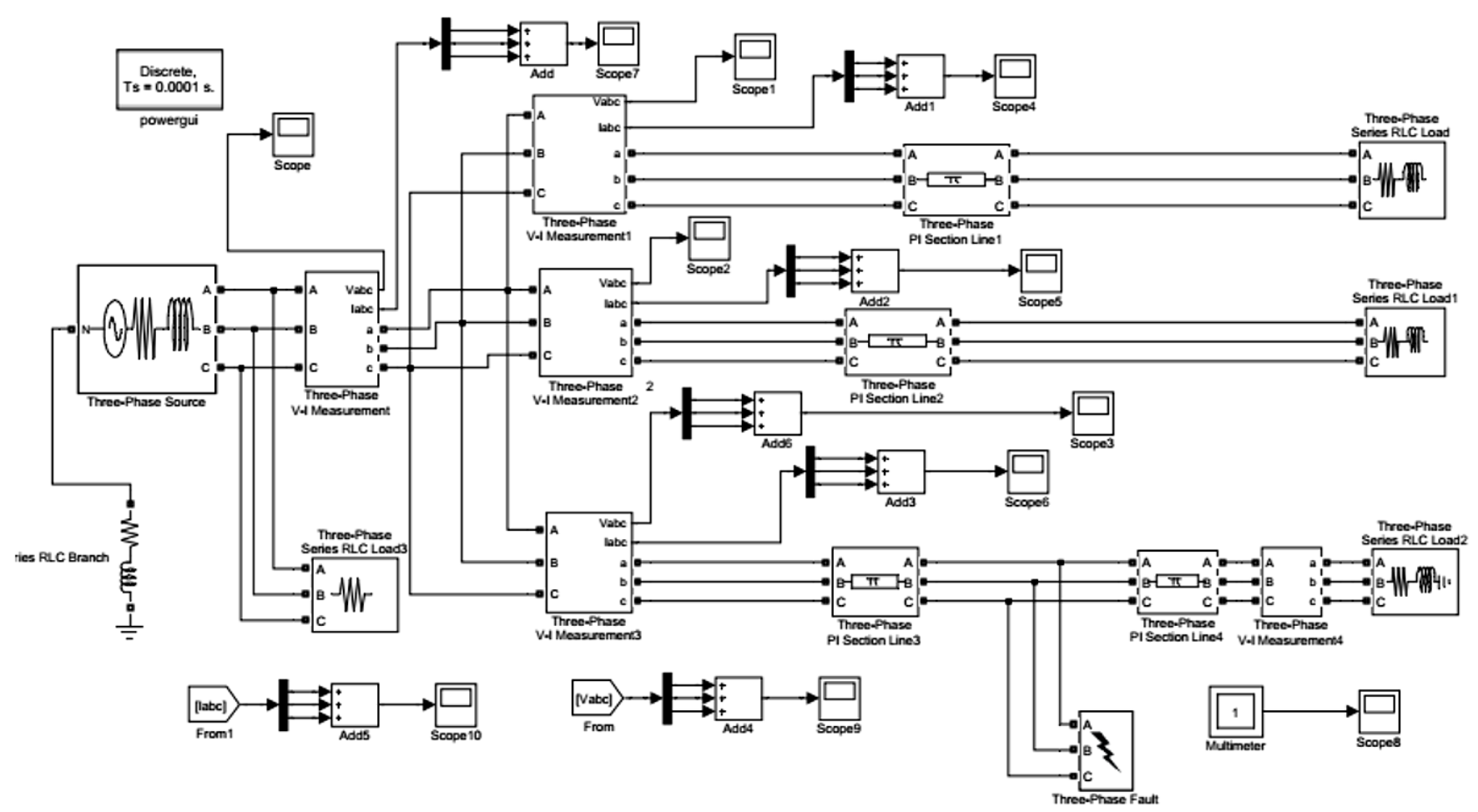

Fig.3 Resonant grounded system simulation diagram

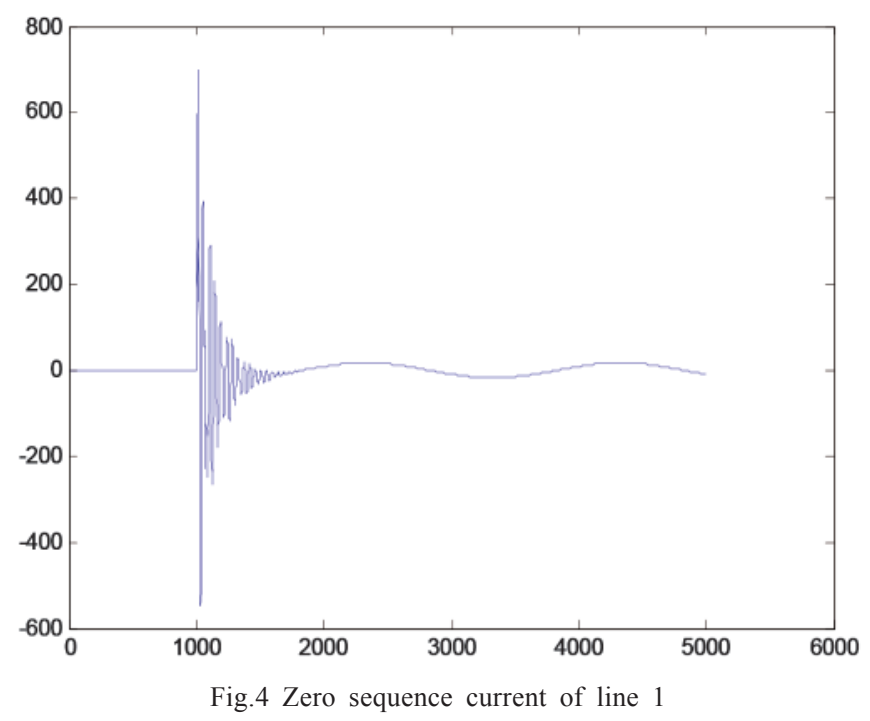

In order to verify the validity of the proposed algorithm, according to the literature [10], the zero sequence current is calculated by HHT and time-frequency amplitude spectrum method to find the fault line. Fig.8 is the time frequency analysis boundary spectrum of non-fault line by HHT, and Fig.9 is the time frequency analysis boundary spectrum of fault line by HHT. It can be seen from the above two figures that the fault line feature obviously exists in IMF1, which verify the validity of the proposed theory.

In all, according to the comparison between Figs. 6 and 7 and Figs. 8 and 9, it can be concluded that fault line selection

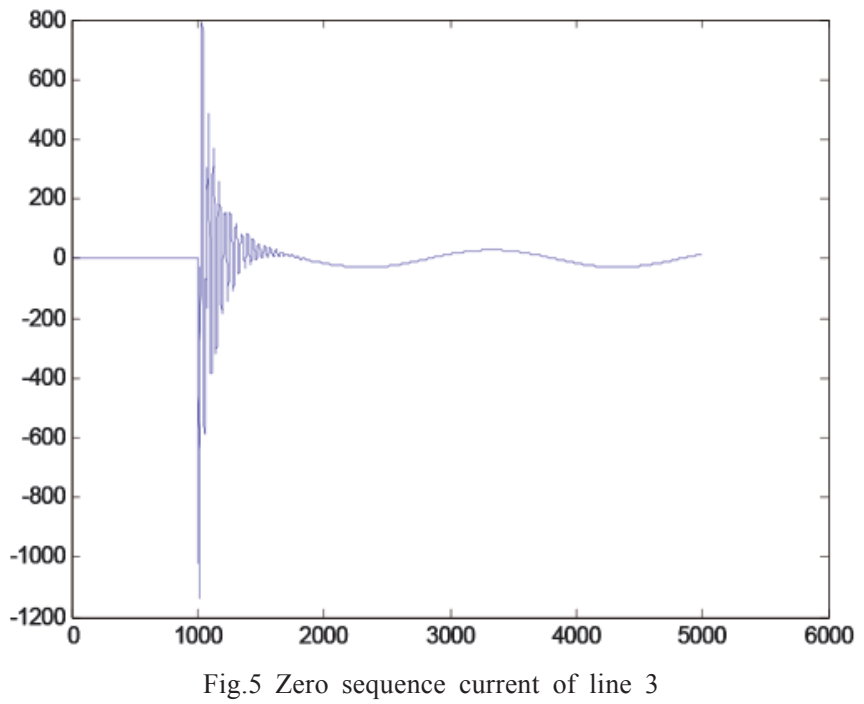

of single phase grounding circuit based on improved HHT is accurate, and the computation time is less than $1 / 10$ of the method mentioned in literature [10].

\subsection{Experimental Data Analysis}

Experimental data: The resonant grounding model is tested in the laboratory. The zero sequence currents of 5 analog circuits system are sampled by $100 \mathrm{~K} / \mathrm{s}$ sampling rate when single-phase ground fault occurs. At the same time, single phase fault data of industrial field are collected by fault recorder in $35 \mathrm{kV}$ substation of DongTan coal mine, and 20 sets of data are collected. 


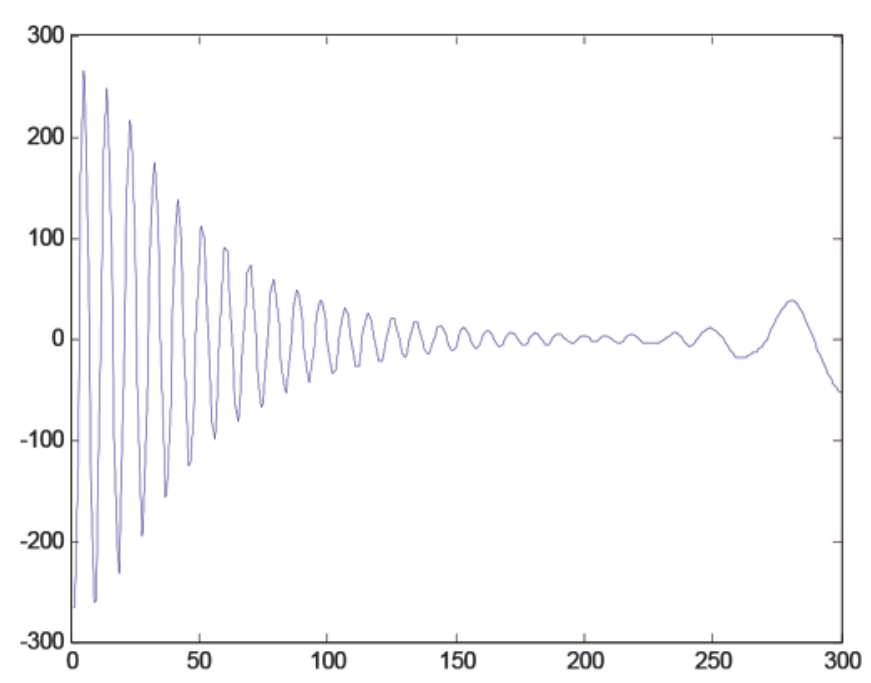

Fig.6 IMF1 of non-fault line



Fig.7 IMF1 of fault line

Data processing method: There are sampling error, quantization error and test line interference error in the experimental data. Zero sequence current is small close to zero before fault occurs, and there is a mutation compared to that of fault line, which can cause HHT end effect and further give rise to the obvious polarity of the former about 5 points of the IMF1 of the fault line and non-fault line. So we can detect the fault line from the former 20 points of the zero sequence

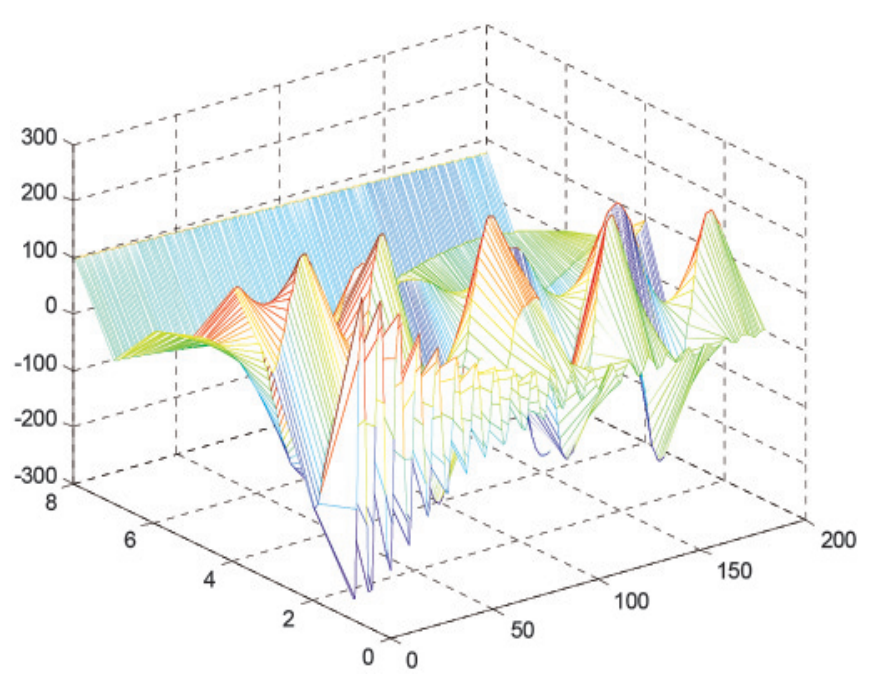

Fig.8 Time frequency analysis boundary spectrum of non-fault line by HHT

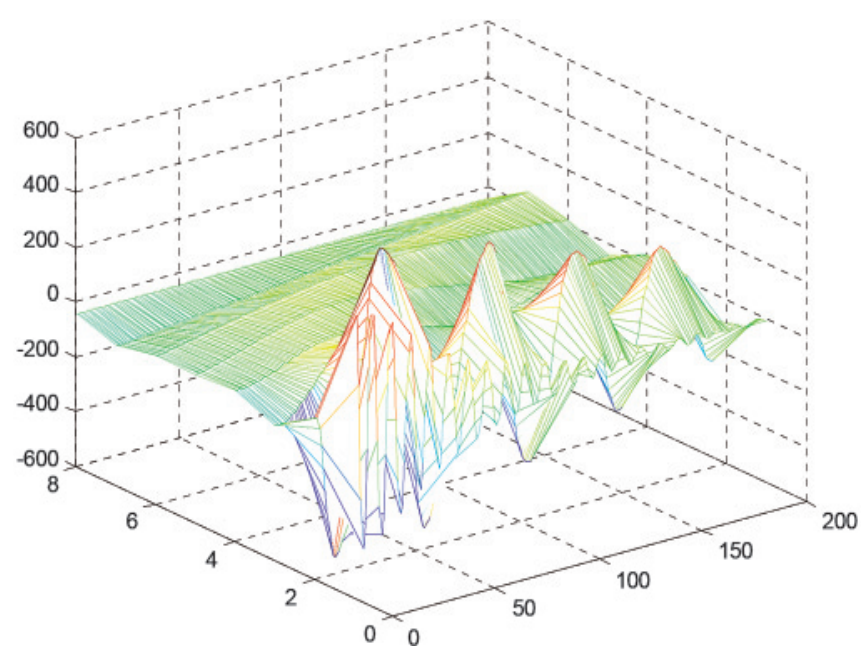

Fig.9 Time frequency analysis boundary spectrum of fault line by HHT

current by use of the improvement of HHT end effect.

Compared algorithm: Wavelet maximum value analysis [4-5], zero sequence fundamental wave analysis, marginal spectrum analysis of HHT[10], IMF1 extraction based on improved HHT proposed in this paper, marginal spectrum analysis based on improved HHT proposed in this paper. And the comparing results are listed in Table 2.

TABLE 2: COMPARISON OF RESULTS OF DIFFERENT ALGORITHMS

\begin{tabular}{lcccc}
\hline $\begin{array}{l}\text { Fault diagnosis } \\
\text { algorithm }\end{array}$ & $\begin{array}{c}\text { Data acquisition } \\
\text { time (millisecond) }\end{array}$ & $\begin{array}{c}\text { Algorithm execution } \\
\text { time (microsecond) }\end{array}$ & $\begin{array}{c}\text { Accuracy } \\
\text { rate }\end{array}$ & $\begin{array}{c}\text { Fault diagnosis } \\
\text { time (millisecond) }\end{array}$ \\
\hline Zero sequence fundamental wave analysis & 40 & $<1$ & 25 & 40 \\
Wavelet maximum value analysis & 10 & $200 \sim 600$ & 23 & $<10.6$ \\
Marginal spectrum analysis of HHT & 10 & $100-400$ & 25 & $<2$ \\
IMF1 extraction based on improved HHT & 10 & $10-200$ & 25 & $<5.001$ \\
Marginal spectrum analysis based on improved HHT & 10 & $<.3$ & 25 \\
\hline
\end{tabular}


Data acquisition time: The time from the failure start to enough data collected for judging the fault.

Algorithm execution time: The time cost by one time fault line selection based on the hardware condition that the computer is $1.2 \mathrm{G}$ processor which is installed with windows $\mathrm{XP}$ operating system and MATLAB7.0 processing environment.

Fault diagnosis time: The time from failure start to the completion of fault line detection.

\subsection{Conclusions}

In this paper, the time-frequency characteristics of the zero sequence current of the single phase grounding in the resonant grounding system and the Hilbert transform are analyzed. We find that system natural oscillation wave contained in the zero sequence current has opposite polarity and greater amplitude in fault line compared to that in nonfault line, and it also has a corresponding relationship with the IMF1 extracted from the same zero sequence current by EMD of HHT. And for EMD, the initial values of cubic spline interpolation function and the fitting data sample can be selected according to the attenuation trend of high frequency oscillation wave. The experimental results show that the improved HHT has the advantages of small computation, fast speed and easy implementation for IMF1 detection and fault line detection, and it can further improve the accuracy of fault detection and reduce the fault line detection time, which has a very important application value in the field.

\subsection{Acknowledgements}

We thank the following organizations for their financial support, namely the Natural Science Foundation of Shandong Province (ZR2014FQ020).

\subsection{References}

[1] Huannian Yao, Meiyue Cao, (2009): "Power System Resonance Grounding” [M], Beijing: China Electric Power Press (The Second Edition), p.21-27

[2] Jing Gong, (2008): "Distribution network integrated automation technology"[M], Beijing: China Machine Press, p.160-183

[3] Hongchun Shu (2008): "The power distribution network fault line selection"[M], Beijing: China Machine Press, p.4-24

[4] Dong Xinzhou, Shi Shenxing (2008): “Identifying
Single-Phase-to-Ground Fault Feeder in Neutral Noneffectively Grounded Distribution System Using Wavelet Transform", IEEE Transactions on Power Delivery, Vol.23(4), p.1829-1937

[5] Yanhui Du (2008): “The Multifractal and Wavelet Theory in the Single-phase Earth Fault Line Selection in Small Current Grounding System Research"[D], Southwest Jiaotong University, p.20-40

[6] Qingle Pang, Shengyin Chen (2009): “The Small Current Grounding Fault Line Selection Based on Artificial Immune Algorithm", Power System Protection and Control, Vol.26(2), p.52-57

[7] Miehalik M., Rebizant W.,Lukowiez M.,et.al. (2006): "High-impedance Fault Detection in Distribution Networks with Use of Wavelet-based Algorithm, IEEE Transaction on power Delivery, Vol.21(4), p.1793-1802

[8] Lai T., Snider L., Lo E.,et. Al. (2005): "Hign-impedance Fault Detection Based on Wavelet Transform and Statistical Pattern Recognition", IEEE Transaction on power Delivery, Vol.20(4), p.2410-2414

[9] Zhigang Liu (2015): "Hilbert-Huang Transform and Its Application in Power System"[M], Science Press, p.79137

[10] Lin Zhang (2013): "Based on the HHT single-phase grounding fault detection research"[D], Hefei University of Technology, p.58-67

[11] Moxin Ju (2015): "Based on the HHT Small Current Grounding Fault Line Selection and Fault Location Method Online"[D], Northeast Electric Power University, p.32-48

[12] Shu Han (2010): "Based on the Hilbert Huang Transform Power System Research of Traveling Wave Fault Location"'[D], Yanshan University, p.28-48

[13] Haishen Zhang,Zhengyou He,Jun Zhang (2012): "Analysis of Characteristics of Resonant Grounding System of Single-phase Grounding Fault Spectrum", Automation of Electric Power Systems, Vol.36(6), p.7881

[14] Na zhao (2008): "HHT Empirical Mode Decomposition Cycle Continuation Method", Computer Simulation, Vol.25(12), p.346-350

[15] Deng Y J,Wang W. (2001): "Boundary Process Technique in EMD Method and Hilbert Transform", Chinese Science Bulletin, Vol.46(11), p.257-263

\section{Indian Journal of Power \& River Valley Development Please renew your subscription}

For details,contact : e-mail: bnjournals@gmail.com 